\title{
UNCERTAINTY AND BIAS IN EXTREME VALUE ANALYSIS OF RECORDS OF STORM SURGES FOR COASTAL PROTECTION PLANS
}

\author{
Toshikazu Kitano, Nagoya Institute of Technology, kitano@nitech.ac.jp
}

\section{MOTIVATIONS}

There are several arguments to be discussed for the probability of hazards due to the storm surge. One is a common point of describing the uncertainty of extreme events, and another point is for the special case due to the storm surge.

1) Return level is one of the important results by extreme value analysis, and the confidence interval also serves us an useful and desirable information for uncertainty. Is it true?

The answer is negative. Return period is right, and important. But the confidence interval, in this case, is shown for return level which is the constant value that is significant after the repeating encounters of the exceedance levels over very long period. But it is of our interest to know which value is the successively occurring level in the future return period, which is a stochastic variable. It is not a constatnt value but unkown even for the God. The prediction interval should be employed for the next realized value of our interest.

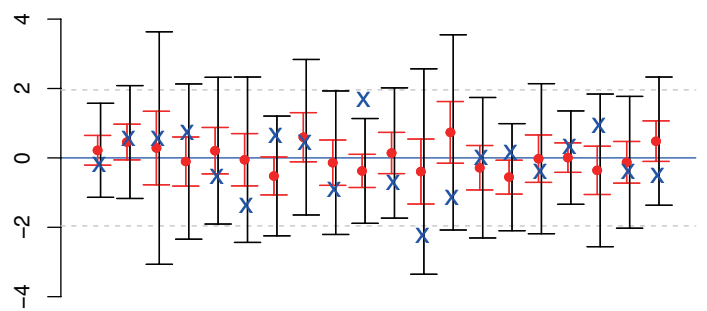

Figure 1 - Two intervals and the actual next observations

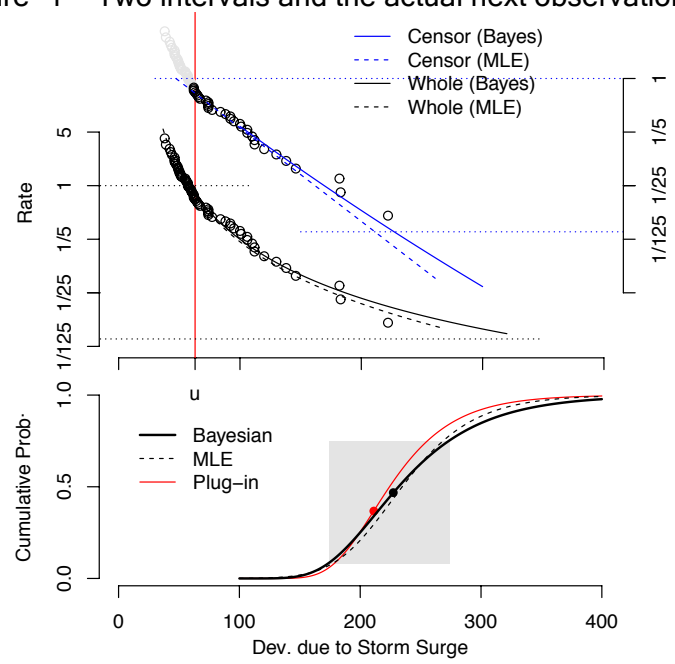

Figure 2 - Estimates for occurrence rate (above); Prediction distribution and the effective range (below)

Fig. 1 shows the prediction intervals (in black) in comparison with the confidence intervals (in red) of the mean for the normal samples. The next realized values (blue $\mathrm{X}$ mark) dose not fall in the confidence intervals in the certain ratio (confidence). This fact is true of extremes. For storm surge data, we show the probability density functions of prediction distribution in Fig. 2 by using the Bayesian method.

2) Is the whole data of annual maximum sea levels valid for the extreme value analysis of storm surge data?

The answer will be NO. Because generally prominant storm surges don't occurr every year in a certain bay. Small values of the annual maximum sea levels will be no good but much harm. Thus, censoring technique works effectively for such problem. Fig. 3 shows that the estimation error of return level (return period $=1 \mathrm{yr}$ ) becomes large as increasing the censoring level while increase of censoring level diminishes the error of return level (return period $100 \mathrm{yrs}$ ). The reason is that censoring can break off the bias due to the unsuitable small values of annual maximum sea levels irrelevant to the storm surge.

\section{COASTAL MANAGEMENT}

Two-stage plan of the protection policies for coastal zone is often considered. Level 1 is the protection by hardware, therefore it is connected directly to the design of sea dikes and breakwaters, and Level 2 is for the evacuation plan, etc. Those stages are generally characterized simply as high/low occurrences of natural hazards, though it cannot convince us of the real scales for the possible phenomena. We believe that the difference of the estimation and prediction will serve the interpretations for the two-stage plan of the protection policies. Our proposal is that the ordinal return period and return level is for Level 1 (high occurrence events) and the upper bund of the prediction range for the maximum over the future time interval for Level 2 (low occurrence events). The representative constant value and the upper limit of the variable are good and original indices for two-stage of natural disaster force.

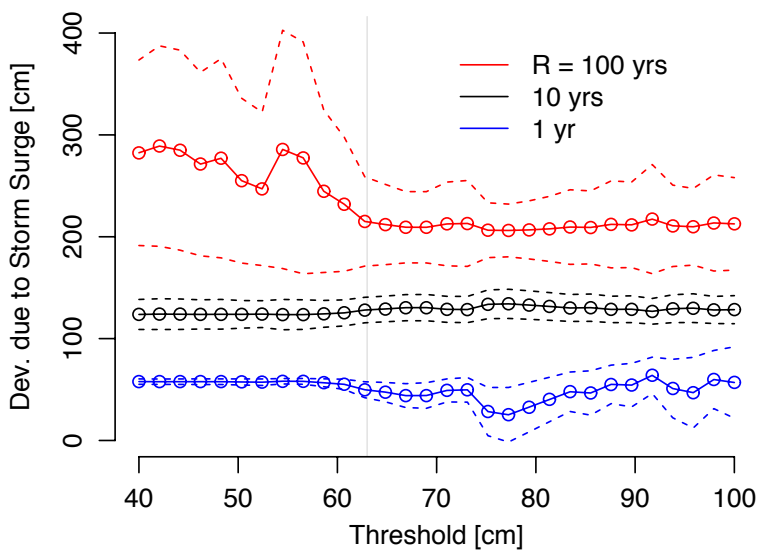

Figure 3 - Changes of error variances by censoring thresholds 